\title{
Table Detection via Probability Optimization
}

\author{
Yalin Wang ${ }^{1}$, Ihsin T. Phillips ${ }^{2}$, and Robert M. Haralick ${ }^{3}$ \\ 1 Dept. of Elect. Eng. Univ. of Washington \\ Seattle, WA 98195, US \\ ylwang@u. washington. edu \\ 2 Dept. of Comp. Science, Queens College, City Univ. of New York \\ Flushing, NY 11367, US \\ yun@image.cs.qc.edu \\ 3 The Graduate School, City Univ. Of New York \\ New York, NY 10016, US \\ haralick@gc. cuny. edu
}

\begin{abstract}
In this paper, we define the table detection problem as a probability optimization problem. We begin, as we do in our previous algorithm, finding and validating each detected table candidates. We proceed to compute a set of probability measurements for each of the table entities. The computation of the probability measurements takes into consideration tables, table text separators and table neighboring text blocks. Then, an iterative updating method is used to optimize the page segmentation probability to obtain the final result. This new algorithm shows a great improvement over our previous algorithm. The training and testing data set for the algorithm include 1,125 document pages having 518 table entities and a total of 10,934 cell entities. Compared with our previous work, it raised the accuracy rate to $95.67 \%$ from $90.32 \%$ and to $97.05 \%$ from $92.04 \%$.
\end{abstract}

\section{Introduction}

With the large number of existing documents and the increasing speed in the production of multitude new documents, finding efficient methods to process these documents for their content retrieval and storage becomes critical. For the last three decades, the document image analysis researchers have successfully developed many outstanding methods for character recognition, page segmentation and understand of text-based documents. Most of these methods were not designed to handle documents containing complex objects, such as tables. Tables are compact and efficient for presenting relational information and most of the documents produced today contain various types of tables. Thus, table structure extraction is an important problem in the document layout analysis field. A few table detection algorithms have been published in the recent literature ( [1]-[2]). However, the performance of these reported algorithms are not yet good enough for commercial usage.

Among the recently published table detection algorithms, some of them are either using a predefined table layout structures [3][4], or relying on complex heuristics for detecting tables ( [5], [6], [7]). Klein et. al. [7] use a signal model to detect tables. Hu et. al. [2] describe an algorithm which detects tables based on computing an optimal partitioning of an input document into some number of tables. They use a dynamic 
programming technique to solve the optimization problem. Their algorithm works for both ASCII and image documents, and according to their global evaluation protocol, the algorithm yields a recall rate of $83 \%$ for 25 ASCII documents and $81 \%$ for 25 scanned images, and a precision rate of $91 \%$ and at $93 \%$, respectively.

Our early table detection work [1] is based on a background analysis, has a coarse to fine hierarchy structure and is a probability based algorithm. It determines the table candidates by finding the large horizontal blank blocks [8] within the document and then statistically validates if the candidates are table entities. Due to the non-iterative nature of this algorithm, its accuracy was not high enough. Figure 1 shows two of failed examples of the earlier algorithm. Figure 1(a) is a false alarm example (b) is a misdetection example.

In this paper, we define the table detection problem as a probability optimization problem. We begin, as we did in our previous algorithm, finding and validating each detected table candidates. We proceed to compute a set of probability measurements for each of the table entities. The computation of the probability measurements takes into consideration tables, table text separators and table neighboring text blocks. Then, an iterative updating method is used to optimize the page segmentation probability to obtain the final result. This new algorithm shows a great improvement over our previous algorithm.

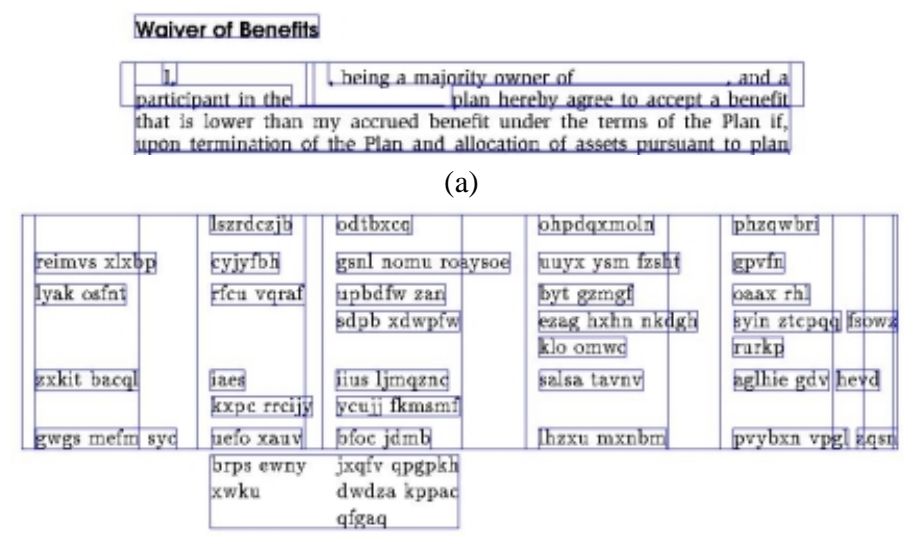

(b)

Fig. 1. Examples of table detection research of our early research; (a) a false alarm example; (b) a misdetection example.

The remainder of the paper is organized as follows. We give the problem statement in Section 2. The probability estimation details are described in Section 3 We present our algorithm details in Section 4. The experimental results are reported in Section 5 and we conclude with our future directions in Section 6 


\section{Table Detection Problem}

Let $\mathcal{A}$ be a set of zone entities. Let $\mathcal{L}$ be a set of content labels, $\{$ table, text-block . Function $f: \mathcal{A} \rightarrow \mathcal{L}$ assigns each element of $\mathcal{A}$ with a label. Function $V: \wp(\mathcal{A}) \rightarrow \Lambda$ computes measurements made on subset of $\mathcal{A}$, where $\Lambda$ is the measurement space.

We define a probability of labeling and measurement function as

$$
P(V(\tau): \tau \in \mathcal{A}, f \mid \mathcal{A})=P(V(\tau): \tau \in \mathcal{A}, \mid f, \mathcal{A}) P(f \mid \mathcal{A})
$$

By making the assumption of conditional independence that when the label $f_{\tau}, \tau \in$ $\mathcal{A}$ is known, no knowledge of other labels will alter the probability of $V(\tau)$, we can decompose the probability in Equation 1 into

$$
P(V(\tau): \tau \in \mathcal{A} \mid f, \mathcal{A})=\underbrace{\prod_{\tau \in \mathcal{A}} P(V(\tau) \mid f, \mathcal{A})}_{(a)} \underbrace{P(f \mid \mathcal{A})}_{(b)}
$$

Expression (a) in Equation 2 can be computed by applying different measurement functions $V_{T A B}$ and $V_{T X T}$ according to $f$ function values, table or text-block, where $V_{T A B}$ is used for tables and $V_{T X T}$ is used for text-blocks.

$$
P(V(\tau): \tau \in \mathcal{A} \mid f, \mathcal{A})=\prod_{f_{\tau}=\text { table }}^{\tau \in \mathcal{A}} P\left(V_{T A B}(\tau) \mid f, \mathcal{A}\right) \prod_{f_{\tau}=\text { text-block }}^{\tau \in \mathcal{A}} P\left(V_{T X T}(\tau) \mid f, \mathcal{A}\right) P(f \mid \mathcal{A})
$$

To compute expression (b) in Equation 2, we consider the discontinuity property between neighbors to two zone entities with different labels. Let $\mathcal{A}=\left\{A_{1}, A_{2}, \cdots, A_{M}\right\}$ be the set of document elements extracted from a document page. Each element $A_{i} \in \mathcal{A}$ is represented by a bounding box $(x, y, w, h)$, where $(x, y)$ is the coordinate of top-left corner, and $w$ and $h$ are the width and height of the bounding box respectively. The spatial relations between two adjacent boxes are shown in Figure 2

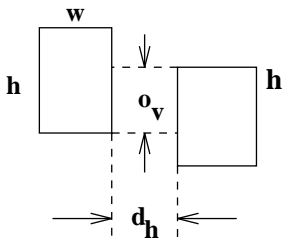

(a)

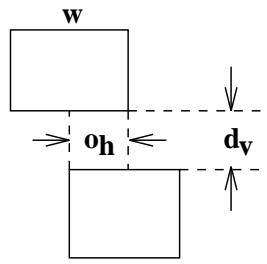

(b)

Fig. 2. Illustrates the spatial relations between two bounding boxes that are (a) horizontally adjacent (b) vertically adjacent. 
For a pair of bounding boxes $a\left(x_{a}, y_{a}, w_{a}, h_{a}\right)$ and $b\left(x_{b}, y_{b}, w_{b}, h_{b}\right)$, the horizontal distance $d_{h}(a, b)$ and vertical distance $d_{v}(a, b)$ between them are defined as

$$
\begin{array}{r}
d_{h}(a, b)= \begin{cases}x_{b}-x_{a}-w_{a} & \text { if } x_{b}>x_{a}+w_{a} \\
x_{a}-x_{b}-w_{b} & \text { if } x_{a}>x_{b}+w_{b} \\
0 & \text { otherwise }\end{cases} \\
d_{v}(a, b)= \begin{cases}y_{b}-y_{a}-h_{a} & \text { if } y_{b}>y_{a}+h_{a} \\
y_{a}-y_{b}-h_{b} & \text { if } y_{a}>y_{b}+h_{b} \\
0 & \text { otherwise }\end{cases}
\end{array}
$$

The horizontal overlap $o_{h}(a, b)$ and vertical overlap $o_{v}(a, b)$ between $a$ and $b$ are defined as

$$
\begin{array}{r}
o_{h}(a, b)= \begin{cases}x_{a}+w_{a}-x_{b} & \text { if } x_{b}>x_{a}, x_{b}<x_{a}+w_{a} \\
x_{b}+w_{b}-x_{a} & \text { if } x_{a}>x_{b}, x_{a}<x_{b}+w_{b} \\
0 & \text { otherwise }\end{cases} \\
o_{v}(a, b)= \begin{cases}y_{a}+h_{a}-y_{b} & \text { if } y_{b}>y_{a}, y_{b}<y_{a}+h_{a} \\
y_{b}+h_{b}-y_{a} & \text { if } y_{a}>y_{b}, y_{a}<y_{b}+h_{b} \\
0 & \text { otherwise }\end{cases}
\end{array}
$$

Let $A_{a}=\left(x_{a}, y_{a}, w_{a}, h_{a}\right)$ and $A_{b}=\left(x_{b}, y_{b}, w_{b}, h_{b}\right)$ be two zone entities.

- We define $A_{b}$ as a right neighbor of $A_{a}$ if $A_{b} \neq A_{a}, x_{b}>x_{a}$, and $o_{v}(a, b)>0$. Let $B_{a}$ be the set of right neighbors of $A_{a}$. Zone entities $A_{a}$ and $A_{b}$ are called horizontally adjacent if

$$
A_{b}=\arg \min _{A_{i} \in B_{a}}\left(d_{h}(a, i) \mid x_{i}>x_{a}, o_{v}(a, i)>0\right) .
$$

- We define $A_{b}$ as a lower neighbor of $A_{a}$ if $A_{b} \neq A_{a}, y_{b}>y_{a}$, and $o_{h}(a, b)>0$. Let $B_{a}$ be the set of right neighbors of $A_{a}$. Zone entities $A_{a}$ and $A_{b}$ are called vertically adjacent if

$$
A_{b}=\arg \min _{A_{i} \in B_{a}}\left(d_{v}(a, i) \mid y_{i}>y_{a}, o_{h}(a, i)>0\right) .
$$

The neighbor set is defined as

$$
\mathcal{N}=\left\{\left(v_{a}, v_{b}\right) \mid v_{a} \text { and } v_{b} \text { horizontally or vertically adjacent, } v_{a} \in \mathcal{V}, v_{b} \in \mathcal{V}\right\}
$$

Assuming the conditional independence between each neighborhood relationship, expression (b) in Equation 2 can be computed as

$$
P(f \mid \mathcal{A})=\prod_{\{p, q\} \in \mathcal{N}} P_{\{p, q\}}\left(f_{p}, f_{q} \mid p, q\right)
$$

where $P_{\{p, q\}}\left(f_{p}, f_{q} \mid p, q\right)$ has the property

$$
P_{\{p, q\}}\left(f_{p}, f_{q} \mid p, q\right)= \begin{cases}P_{\{p, q\}}\left(f_{p}, f_{q} \mid p, q\right) & f_{p} \neq f_{q} \\ 0 & f_{p}=f_{q}\end{cases}
$$


Equation 3 can be written as

$$
\begin{aligned}
& P(V(\tau): \tau \in \mathcal{A} \mid f, \mathcal{A})= \\
& \quad \prod_{f_{\tau}=\text { table }}^{\tau \in \mathcal{A}} P\left(V_{T A B}(\tau) \mid f, \mathcal{A}\right) \prod_{f_{\tau}=\text { text-block }}^{\tau \in \mathcal{A}} P\left(V_{T X T}(\tau) \mid f, \mathcal{A}\right) \prod_{\{p, q\} \in \mathcal{N}} P\left(f_{p}, f_{q} \mid p, q\right)
\end{aligned}
$$

The table detection problem can be formulated as follows: Given initial set $\mathcal{A}^{0}$, find a new set $\mathcal{A}^{s}$ and a labeling function $f^{s}: A^{s} \rightarrow L$, that maximizes the probability:

$$
\begin{aligned}
& P\left(V(\tau): \tau \in \mathcal{A}^{s} \mid f^{s}, \mathcal{A}^{s}\right)= \\
& \quad \prod_{f_{\tau}^{s}=\text { table }}^{\tau \in \mathcal{A}^{s}} P\left(V_{T A B}(\tau) \mid f^{s}, \mathcal{A}^{s}\right) \prod_{f_{\tau}^{s}=t e x t-\text { block }}^{\tau \in \mathcal{A}^{s}} P\left(V_{T X T}(\tau) \mid f^{s}, \mathcal{A}^{s}\right) \prod_{\{p, q\} \in \mathcal{N}} P\left(f_{p}^{s}, f_{q}^{s} \mid p, q\right)
\end{aligned}
$$

Our goal is to maximize the probability in Equation 12 by iteratively updating $\mathcal{A}^{k}$ and $f^{k}$. Our table detection system works as follows: we used our early research [1] to get preliminary table detection results. Then we systematically adjust the labeling to maximize the probability until no further improvement can be made.

\section{Probability Estimation}

\subsection{Table and Text Separator Probability}

Given a table, $t$ and its vertically adjacent neighboring text block $B$, we compute the probability of the separator between them being a table and text separator as

$$
P\left(f_{t}, f_{B} \mid t, B\right)=P\left(\text { TableTextSeparator } \mid o_{h}(t, B), d_{v}(t, B)\right)
$$

where the definitions of $d_{v}(t, B)$ and $o_{h}(t, B)$ can be found at Equation 5 and Equation 6 .

\subsection{Table Measurement Probability}

To facilitate table detection, we applied our table decomposition algorithm [1] on each detected table. Based on the table decomposition results, three features are computed. These features are given below.

- Ratio of total large vertical blank block [8] and large horizontal blank block [8] areas over identified table area. Let $t$ be an identified table and $\mathcal{B}$ be the set of large horizontal and vertical blank blocks and in it, $r a=\frac{\sum_{\beta \in \mathcal{B}} \text { Area }(\beta)}{\text { Area }(t)}$;

- Maximum difference of the cell baselines in a row. Denote the set of the cells in a row $i$ as $\mathcal{R C}_{i}, \mathcal{R C}_{i}=\left\{c_{i, 1}, c_{i, 2}, \ldots, c_{i, i_{m}}\right\}$. Denote the set of $\mathcal{R C}_{i}$ as $\mathcal{R C}, \mathcal{R C}=$ $\left\{\mathcal{R C}_{i}, i=1, \ldots, m\right\}$, where $m$ is the row number in the table. Let baseline $(c)$ be the $y$ coordinate of the cell entity bottom line, $m c=\underset{\mathcal{R C}_{i} \in \mathcal{R} \mathcal{C}}{\max }\left(\underset{c_{i, j} \in \mathcal{R C}_{i}}{\max }\left(\operatorname{baseline}\left(c_{i, j}\right)\right)-\right.$ $\left.\min _{c_{i, j} \in \mathcal{R C}_{i}}\left(\operatorname{baseline}\left(c_{i, j}\right)\right)\right)$; 
- Accumulated difference of the justification in all columns. Denote the set of cells in a column, $i$, in the table $\mathcal{C C}_{i}=\left\{c_{i, 1}, c_{i, 2}, \ldots, c_{i, i_{n}}\right\}$. Denote the set of $\mathcal{C C}_{i}$ as $\mathcal{C C}, \mathcal{C C}=\left\{\mathcal{C C}_{i}, i=1, \ldots, n\right\}$, where $n$ is the column number in the table. Let $x_{i, j}, y_{i, j}, w_{i, j}, h_{i, j}$ represent the bounding box of the cell $c_{i, j} \in \mathcal{C C}_{i}$. We estimate the justification of a column, $i, i=1, \ldots, n$, by computing the vertical projection of the left, center, and right edge of $c_{i, j}, j=1, \ldots, i_{n}$,

$$
\begin{aligned}
C_{\text {left }}[i] & =\max _{c_{i, j} \in \mathcal{C C}_{i}}\left(x_{i, j}\right)-\min _{c_{i, j} \in \mathcal{C C}_{i}}\left(x_{i, j}\right) \\
C_{\text {center }}[i] & =\max _{c_{i, j} \in \mathcal{C C}_{i}}\left(x_{i, j}+w_{i, j} / 2\right)-\min _{c_{i, j} \in \mathcal{C C}_{i}}\left(x_{i, j}+w_{i, j} / 2\right) \\
C_{\text {right }}[i] & =\max _{c_{i, j} \in \mathcal{C C}_{i}}\left(x_{i, j}+w_{i, j}\right)-\min _{c_{i, j} \in \mathcal{C C}_{i}}\left(x_{i, j}+w_{i, j}\right) \\
J_{i} & =\min \left\{C_{\text {left }}[i], C_{\text {center }}[i], C_{\text {right }}[i]\right\}
\end{aligned}
$$

The accumulated difference of the justification in all columns, $m j$, is computed as: $m j=\sum_{i=1}^{n} J_{i}$.

Finally, we can compute the table consistent probability for table $t$ as

$$
P\left(V_{T A B}(t)\right)=P(\text { consistency }(t) \mid r a(t), m c(t), m j(t))
$$

\subsection{Text Block Measurement Probability}

A text block, in general, has a homogeneous inter-line spacing and an alignment type (such as left-justisfied, etc.) Given a detected text block $B$, we compute the probability that $B$ has homogeneous inter-line spacing, and a text alignment type. We define leading as inter-line spacing. As in Liang et. al.' [9], we compute text block measurement probability as

$$
P\left(V_{T X T}(B)\right)=P\left(V_{T X T}(B) \mid L e a d i n g(B), \text { Alignment }(B)\right)
$$

By making the assumption of conditional independence, we can rewrite the above equation as

$$
P\left(V_{T X T}(B)\right)=P\left(V_{T X T}(B) \mid \operatorname{Leading}(B)\right) P\left(V_{T X T}(B) \mid \operatorname{Alignment}(B)\right)
$$

Let $B=\left(l_{1}, \ldots, l_{n}\right)$ be an extracted block. $D_{B}=(d(1), d(2), \ldots, d(n-1))$ is a sequence of inter-line space distances, where $d(j)$ is the space distance between $l_{j}$ and $l_{j+1}$. We compute the median and the maximum value of the elements of $D_{B}$. The probability is

$$
P\left(V_{T X T}(B) \mid L e a d i n g(B)\right)=P\left(\operatorname{median}\left(D_{B}\right), \max \left(D_{B}\right) \mid \text { Leading }(B)\right) .
$$

Given a text block $B$ that consists of a group of text lines $B=\left(l_{1}, l_{2}, \cdots, l_{n}\right)$, we determine the text alignment of $B$ by observing the alignment of the text line edges. Let $e_{l i}$ be the left edge of the text line $l_{i}$ and let $e_{c i}$ and $e_{r i}$ be the center and right edges of the line box respectively. Let $E_{l}$ be the left edges of text line 2 to $n$, such that $E_{l}=\left\{e_{l i} \mid 2 \leq i \leq n\right\} . E_{c}$ is the center edges of text line 2 to $n-1$, and $E_{r}$ is the 
right edges of text line 1 to $n-1$. We first estimate the median of $E_{l}$, then compute the absolute deviation $D_{l}$ of the elements of $E_{l}$ from its median,

$$
D_{l}=\left\{d_{i}\left|d_{i}=\right| e_{l i}-\operatorname{median}\left(E_{l}\right) \mid, 2 \leq i \leq n\right\} .
$$

Similarly, we estimate the absolute deviation of the center edges and right edges: $D_{c}$ and $D_{r}$. Then, we compute the probability of $B$ being left, center, right, or both justified by observing the mean absolute deviation of the left, center and right edges,

$$
P\left(V_{T X T}(B) \mid \operatorname{Alignment}(B)\right)=P\left(\operatorname{mean}\left(D_{l}\right), \text { mean }\left(D_{c}\right), \text { mean }\left(D_{r}\right) \mid \text { Alignment }(B)\right) .
$$

\section{Table Detection Algorithm}

Figure 3 shows our algorithm diagram. Given a labeled page, first we estimate its segmentation probability. For each table, we consider several adjustments, which are to keep it as a table, to grow the table to include its upper and lower neighbors, to merge the table with its upper and lower neighbors and label it as text block. For each adjustment, we compute the new probability. We select the adjustment which produces the biggest improvement upon the initial page segmentation probability. This process is repeated until no improvement can be made. The details of the algorithm are described below.

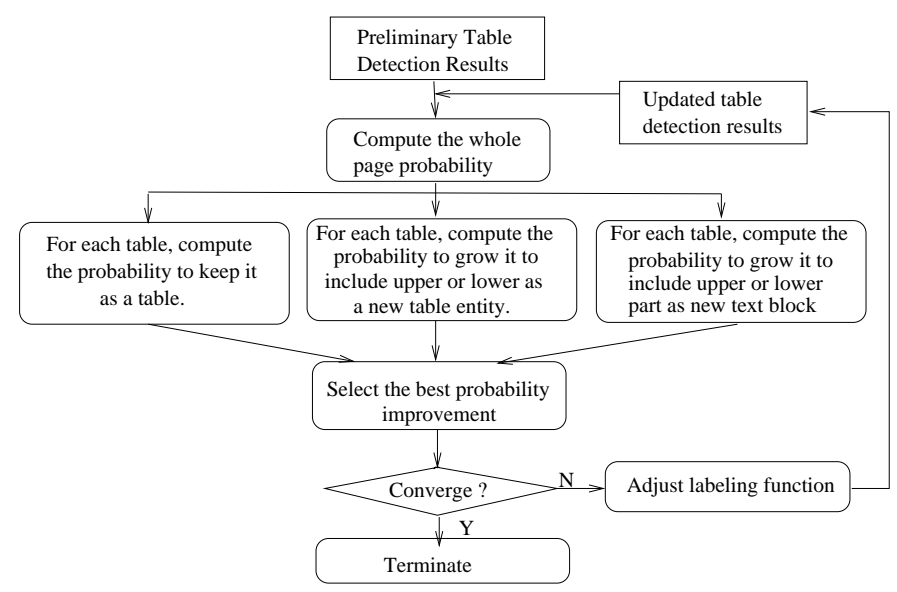

Fig. 3. Overview of the table detection algorithm

\section{Algorithm 41 Table Optimization}

1. The input data to the algorithm are our previous table detection [1] and text block segmentation results. They are a set of block entities, $\mathcal{A}^{0}$ and function $f^{0}: \mathcal{A}^{0} \rightarrow \mathcal{L}$; 
2. Set $k=0$;

3. For each hypothesized table, $i, i=1, \ldots, N$, where $N$ is the number of tables in $\mathcal{A}^{k}$. Compute the different probabilities under different adjustments.

- Keep the table. Compute the probability $P_{(i, 1)}$ following Equation 12,

- Merge table $i$ with its upper text neighbor and label it as a new table. Compute the new probability $P_{(i, 2)}$ following Equation 12

- Merge table $i$ with its upper text neighbor and label it as a new text block. Compute the new probability $P_{(i, 3)}$ following Equation 12 .

- Merge table $i$ with its lower text neighbor and label it as a new table. Compute the new probability $P_{(i, 4)}$ following Equation 12

- Merge table $i$ with its lower text neighbor and label it as a new text block. Compute the new probability $P_{(i, 5)}$ following Equation 12 .

4. Compute $P_{\max }=\max \left(P_{(i, j)}\right), i=1, \ldots, N, j=1, \ldots, 5$ and get its appropriate adjustment action.

5. If the action is to keep the table, then, return the labeling result $\mathcal{A}^{k}$ as $\mathcal{A}^{s}$ and stop the algorithm.

6. If the action is not to keep the table, then take the adjustment action and we get $\mathcal{A}^{k+1}$ and $f^{k+1}: \mathcal{A}^{k+1} \rightarrow \mathcal{L}$.

7. Set $k=k+1$ and go back to 3 ,

\section{Experimental Results}

Our test data set has 1, 125 document pages [1]. All of them are machine printed, noise free data. Among them, 565 pages are real data from different business and law books. Another 560 pages are synthetic data generated using the method described in [1]. A holdout cross validation experiment [10] was conducted on all the data with $N=9$. Discrete lookup tables were used to represent the estimated joint and conditional probabilities used at each of the algorithm decision steps.

Suppose we are given two sets $\mathcal{G}=\left\{G_{1}, G_{2}, \ldots, G_{M}\right\}$ for ground-truthed foreground table related entities, e.g. cell entities, and $\mathcal{D}=\left\{D_{1}, D_{2}, \ldots, D_{N}\right\}$ for detected table related entities. The algorithm performance evaluation can be done by solving the correspondence problem between the two sets. Performance metrics developed in [11] can be directly computed in each rectangular layout structure set. The performance evaluation was done on the cell level. The numbers and percentages of miss, false, correct, splitting, merging and spurious detections on real data set and on the whole data set are shown in Table 1 and Table 2 respectively. Compared with our early work [1], we improved the detection result rates from $90.32 \%$ to $95.67 \%$ and from $92.04 \%$ to $97.05 \%$ in the whole data set. For the real data set, we improved the detection result rates from $89.69 \%$ to $96.76 \%$ and from $93.12 \%$ to $93.86 \%$.

Figure 4 shows a few table detection examples. Figure 4(a), (b) are the correct detection results of Figure 1(a), (b), respectively. In Figure 4(a), our algorithm grows the original table and include its lower neighbor and construct a new table entity. In Figure 4(b), our algorithm eliminates the originally detected table and merge it with its lower neighbor and construct a new text block entity. Figure 4(c), (d) illustrate some failed 


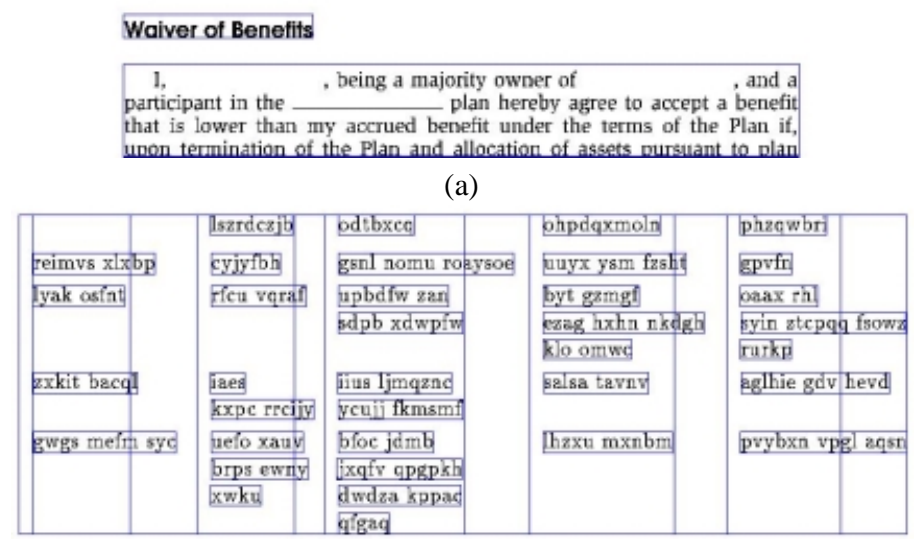

(b)

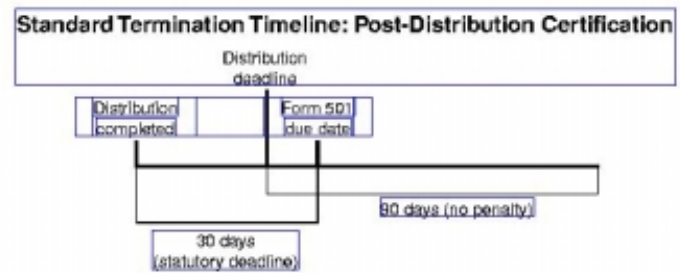

(c)

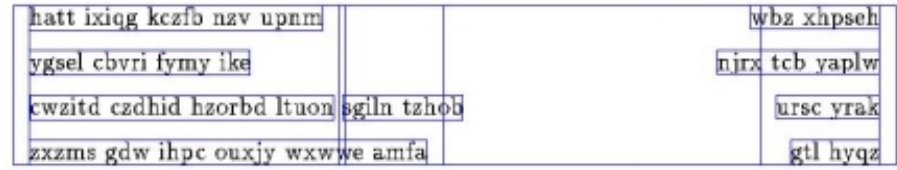

(d)

Fig. 4. Illustrates the table detection results; (a), (b) Correct table detection results; (c), (d) failed table detection results.

examples. Figure 4(c) shows a false alarm example. Some texts in a figure are detected as a table entity. Figure 4(d) shows an error example where our table decomposition algorithm failed.

\section{Conclusion and Future Work}

In this paper, we formulated table detection problem in the whole page segmentation framework. We tried to improve table detection result by optimizing the whole page segmentation probability, including table entities, text block entities and the separators between them. We used iterative updating method to improve the probability. We implemented our algorithm and tested on a data set which includes 1, 125 document pages with 10,934 table cell entities. Among them, 565 pages are real data from differ- 
Table 1. Cell level performance of the table detection algorithm on real data set.

\begin{tabular}{|r|r|r|r|r|r|r|}
\hline & Total & Correct & Splitting & Merging & Mis-False & Spurious \\
\hline Ground Truth & 679 & 657 & 3 & 15 & 4 & 0 \\
& & $(96.76 \%)$ & $(0.44 \%)$ & $(2.21 \%)$ & $(0.59 \%)$ & $(0.00 \%)$ \\
\hline Detected & 700 & 657 & 6 & 7 & 30 & 0 \\
& & $(93.86 \%)$ & $(0.86 \%)$ & $(1.00 \%)$ & $(4.29 \%)$ & $(0.00 \%)$ \\
\hline
\end{tabular}

Table 2. Cell level performance of the table detection algorithm on the whole data set.

\begin{tabular}{|r|r|r|r|r|r|r|}
\hline & Total & Correct & Splitting & Merging & Mis-False & Spurious \\
\hline Ground Truth & 10934 & 10461 & 132 & 45 & 296 & 0 \\
& & $(95.67 \%)$ & $(1.21 \%)$ & $(0.41 \%)$ & $(2.71 \%)$ & $(0.00 \%)$ \\
\hline Detected & 10779 & 10461 & 264 & 18 & 36 & 0 \\
& & $(97.05 \%)$ & $(2.45 \%)$ & $(0.17 \%)$ & $(0.33 \%)$ & $(0.00 \%)$ \\
\hline
\end{tabular}

ent business and law books. Another 560 pages are synthetic data generated using the method described in [1]. The experimental results demonstrated the improvement of our algorithm.

As shown in Figure 4(d), our current table decomposition algorithm needs further refined, incorporating some additional information carrying features. Our formulation has the potential to be useful to other more general page segmentation problems, for example, to segment text, figure and images, etc. We will study this in the future.

\section{References}

1. Y. Wang, I. T. Phillips, and R. Haralick. Automatic table ground truth generation and a background-analysis-based table structure extraction method. In Sixth International Conference on Document Analysis and Recognition(ICDAR01), pages 528-532, Seattle, WA, September 2001.

2. J. Hu, R. Kashi, D. Lopresti, and G. Wilfong. Medium-independent table detection. In SPIE Document Recognition and Retrieval VII, pages 291-302, San Jose, California, January 2000.

3. E. Green and M. Krishnamoorthy. Model-based analysis of printed tables. In Proceedings of the 3rd ICDAR, pages 214-217, Canada, August 1995.

4. J. H. Shamilian, H. S. Baird, and T. L. Wood. A retargetable table reader. In Proceedings of the 4th ICDAR, pages 158-163, Germany, August 1997.

5. T. G. Kieninger. Table structure recognition based on robust block segmentation. Document Recognition V., pages 22-32, January 1998.

6. T. Kieninger and A. Dengel. Applying the t-rec table recognition system to the business letter domain. In Sixth International Conference on Document Analysis and Recognition(ICDAR01), pages 518-522, Seattle, WA, September 2001.

7. B. Klein, S. Gokkus, T. Kieninger, and A. Dengel. Three approaches to "industrial" table spotting. In Sixth International Conference on Document Analysis and Recognition(ICDAR01), pages 513-517, Seattle, WA, September 2001. 
Y. Wang, I.T. Phillips, and R.M. Haralick

8. Y. Wang, R. Haralick, and I. T. Phillips. Improvement of zone content classification by using background analysis. In Fourth IAPR International Workshop on Document Analysis Systems. (DAS2000), Rio de Janeiro, Brazil, December 2000.

9. J. Liang, I. T. Phillips, and R. M. Haralick. Consistent partition and labeling of text blocks. Journal of Pattern Analysis and Applications, 3:196-208, 2000.

10. R. Haralick and L. Shapiro. Computer and Robot Vision, volume 1. Addison Wesley, 1997.

11. J. Liang. Document Structure Analysis and Performance Evaluation. Ph.D thesis, Univ. of Washington, Seattle, WA, 1999. 